Article

\title{
Pulse Microcalorimetry Study of Methane Dry Reforming Reaction on Ni/Ceria-Zirconia Catalyst
}

\author{
Mikhail N. Simonov ${ }^{1,2}$ (D), Vladimir A. Rogov ${ }^{1,2}$, Marina Yu. Smirnova ${ }^{1,2}$ and \\ Vladislav A. Sadykov 1,2,* \\ 1 Boreskov Institute of Catalysis, Prospekt Akademika Lavrentieva, 5, Novosibirsk 630090, Russia; \\ smike@catalysis.ru (M.N.S.); rogov45@mail.ru (V.A.R.); smirnova@catalysis.ru (M.Y.S.) \\ 2 Novosibirsk State University, Pirogova Street, 2, Novosibirsk 630090, Russia \\ * Correspondence: sadykov@catalysis.ru; Tel.: +7-383-330-8767
}

Received: 24 August 2017; Accepted: 7 September 2017; Published: 12 September 2017

\begin{abstract}
For Ni/CeZrO catalyst prepared in supercritical isopropanol main features of methane dry reforming reaction mechanism were studied by the pulse microcalorimetric technique. The reaction scheme is described by a step-wise redox mechanism with independent stages of $\mathrm{CH}_{4}$ transformation on $\mathrm{Ni}$ /support interface producing syngas with participation of support oxygen bridging species (the rate-limiting stage) and fast reoxidation of support sites by $\mathrm{CO}_{2}$ yielding $\mathrm{CO}$ regenerating reactive oxygen species.
\end{abstract}

Keywords: synthesis in supercritical alcohol; ceria-zirconia; pulse microcalorimetry; methane dry reforming; mechanism

\section{Introduction}

Catalysts consisting of metal nanoparticles $(\mathrm{Pt}, \mathrm{Ru}, \mathrm{Ni}+\mathrm{Ru})$ deposited on the surface of fluorite-like ( $\mathrm{Ln}-\mathrm{Ce}-\mathrm{Zr}-\mathrm{O}$ ) oxides with high surface/lattice oxygen mobility and reactivity demonstrate high activity and stability to coke formation in dry methane reforming [1-5]. This is explained by the bifunctional scheme of the reaction mechanism including activation of $\mathrm{CH}_{4}$ on metal sites, $\mathrm{CO}_{2}$-on oxide sites with the subsequent rapid transfer of oxygen species to the metal-support interface, where they interact with activated $\mathrm{CH}_{\mathrm{x}}$ species producing syngas [6]. However, for undoped ceria-zirconia oxides with loaded Ni there is still no direct evidence of such a mechanism, and the question remains what is the nature of the rate determining stage. Another question concerns the activation of reagents on these catalysts being really independent or conjugated. Indeed, for traditional catalysts such as $\mathrm{Pt}, \mathrm{Ni}$, and Co supported on alumina, the pulse microcalorimetric studies by Bychkov et al. $[7,8]$ have reliably demonstrated that $\mathrm{CO}_{2}$ is activated by direct interaction with carbon atoms on the surface of metal, which is the slowest step of dry reforming. For the $\mathrm{Ni} / \mathrm{SiO}_{2}$ catalyst, the TAP (Temporal Analysis of Products) studies of Schuurman and Mirodatos have shown that $\mathrm{CO}_{2}$ is activated by direct dissociation on $\mathrm{Ni}$ atoms, whereas the rate-limiting step is the interaction between $\mathrm{C}$ and $\mathrm{O}$ adsorbed on $\mathrm{Ni}$ [9]. Consequently, depending on the support nature even for the same metal, different ways of $\mathrm{CO}_{2}$ activation can take place. The evaluation of the conversion of reagents in mixed and separate pulses fed to a steady-state catalyst is a conventional approach to elucidate the reaction mechanism of catalytic red-ox reactions [7-13]. However, it has not yet been used to study dry reforming of methane on catalysts consisting of inexpensive Ni deposited on undoped mixed ceria-zirconia oxides with a high uniformity of the spatial distribution of $\mathrm{Ce}$ and $\mathrm{Zr}$ cations providing a high mobility and reactivity of the surface/bulk oxygen. In this work, for Ni/CeZrO catalyst prepared by a novel continuous preparation technique in supercritical alcohol medium [14] pulse microcalorimetric studies were conducted to determine the main features of the reaction mechanism of methane dry reforming. 


\section{Results}

\subsection{Catalyst Characterization}

According to XRD (X-ray diffraction) data presented in [14], the calcined sample represents $\mathrm{CeO}_{2}-\mathrm{ZrO}_{2}$ solid solution of pseudocubic fluorite phase referred to as the Fm3m space group. The spherical-shaped particles of ceria-zirconia catalyst with size of 100-500 nm consist of smaller crystallites with sizes up to $22 \mathrm{~nm}$. Specific surface area of the catalyst is $9 \mathrm{~m}^{2} \mathrm{~g}^{-1}$. For the calcined sample, $\mathrm{NiO}$ nanoparticles with average size ranging from 23 to $28 \mathrm{~nm}$ were revealed according to TEM (Transmission electron microscopy). It is worth noting that these values are somewhat greater than those estimated by X-ray diffraction $(15 \mathrm{~nm})$. This may be caused by the presence of bulk defects in $\mathrm{NiO}$ crystallites resulting in a decrease of the effective X-ray crystallite size.

\subsection{Pulse Microcalorimetry at $973 \mathrm{~K}$}

The temperature of dry reforming of methane was chosen in order to obtain either full (973 K) or nearly a half $(773 \mathrm{~K})$ conversion of methane.

The experimental sequence was as follows:

1. Continuous flow of reaction mixture $\left(5 \% \mathrm{CH}_{4}\right.$ and $\left.\mathrm{CO}_{2}\right)$ in He at $973 \mathrm{~K}$ for $30 \mathrm{~min}$. After that small (pulse volume $0.85 \mathrm{~mL}$ ) probing pulses and big (pulse volume $3.6 \mathrm{~mL}$ ) pulses of reaction mixture were fed into the reactor by turns. Mixtures of products from small pulses were analysed by GC (gas chromatography), whereas products from big pulses were not analysed due to GC column overloading. Big pulses were used to achieve steady-state of the catalyst more quickly. It is important to note that the use of alternating small and big pulses was equivalent to the use of only small pulses, and the achieved steady state of the catalyst depended only on the temperature of the reactor and composition of the pulse.

2. Pulses of $5 \% \mathrm{CH}_{4}$ in $\mathrm{He}$ (pulse volume $0.85 \mathrm{~mL}$ ).

3. Small (pulse volume $0.85 \mathrm{~mL}$ ) probing pulses and big (pulse volume $3.6 \mathrm{~mL}$ ) pulses of reaction mixture.

4. Pulses of $5 \% \mathrm{CO}_{2}$ in $\mathrm{He}$.

5. Small (pulse volume $0.85 \mathrm{~mL}$ ) probing pulses and big (pulse volume $3.6 \mathrm{~mL}$ ) pulses of reaction mixture.

6. Pulses of $\mathrm{CO}$ in He.

Figures 1 and 2 show conversions of methane and $\mathrm{CO}_{2}$, the mass balance as well as ratios of hydrogen and $\mathrm{CO}$, including mole of products per mole of converted methane estimated by GC analysis. It should be noted that the mass balance in the entire experiment was close to unity, which indicates the reliability of the data obtained. During the first pulse of the $\mathrm{RM}$ (reaction mixture $\mathrm{CH}_{4}+\mathrm{CO}_{2}$ ), additional $\mathrm{H}_{2}$ and $\mathrm{CO}_{2}$ super-stoichiometric emissions occurred, associated with the participation of the oxygen of the catalyst, which is in a more oxidized state compared to the stationary state of the catalyst. Starting with the 4th pulse of the RM, the conversion of $\mathrm{CO}_{2}$ stabilizes at the level of 0.9 at full conversion of methane, which indicates the achievement of a steady state of the catalyst in methane dry reforming reaction:

$$
\mathrm{CH}_{4}+\mathrm{CO}_{2}=2 \mathrm{H}_{2}+2 \mathrm{CO}
$$

During supply of methane pulses in the absence of $\mathrm{CO}_{2}$ synthesis gas is produced but the ratio of $\mathrm{H}_{2}$ to $\mathrm{CO}$ was about 2 with the total conversion of methane, which is due to the reaction of formation of synthesis gas with the participation of catalyst oxygen $\mathrm{O}_{\text {cat }}$ in accordance with equation:

$$
\mathrm{CH}_{4}+\mathrm{O}_{\text {cat }}=2 \mathrm{H}_{2}+\mathrm{CO} \text {. }
$$


Then the catalyst was again brought to the stationary state using RM pulses (pulses from 13 to 16) and then pulses of $\mathrm{CO}_{2}$ were fed, during which a decrease in the degree of $\mathrm{CO}_{2}$ conversion, characterizing the process of reoxidation of the stationary-state catalyst was observed.

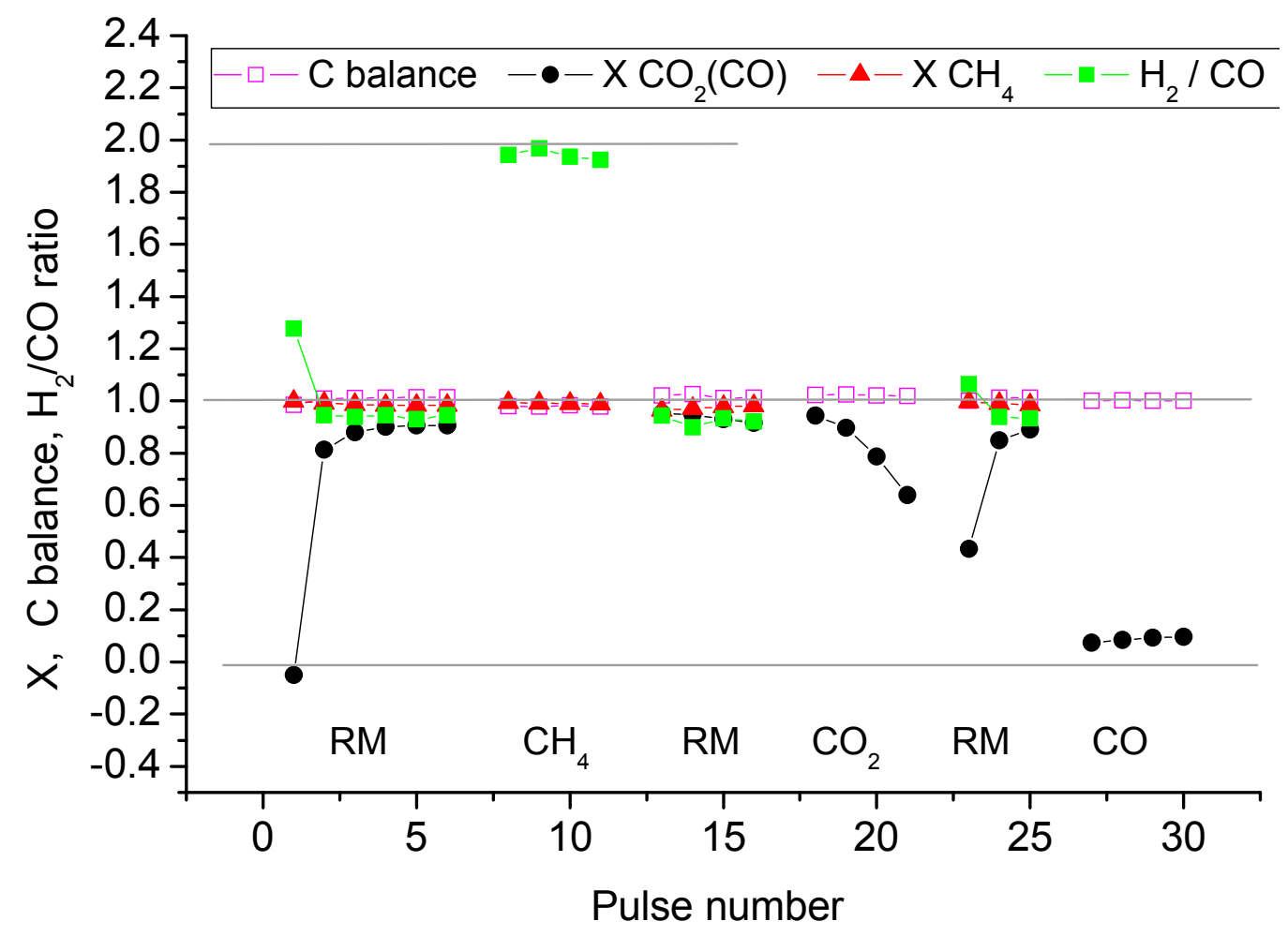

Figure 1. Conversions of reagents and products characteristics in series of pulses at $973 \mathrm{~K}$.

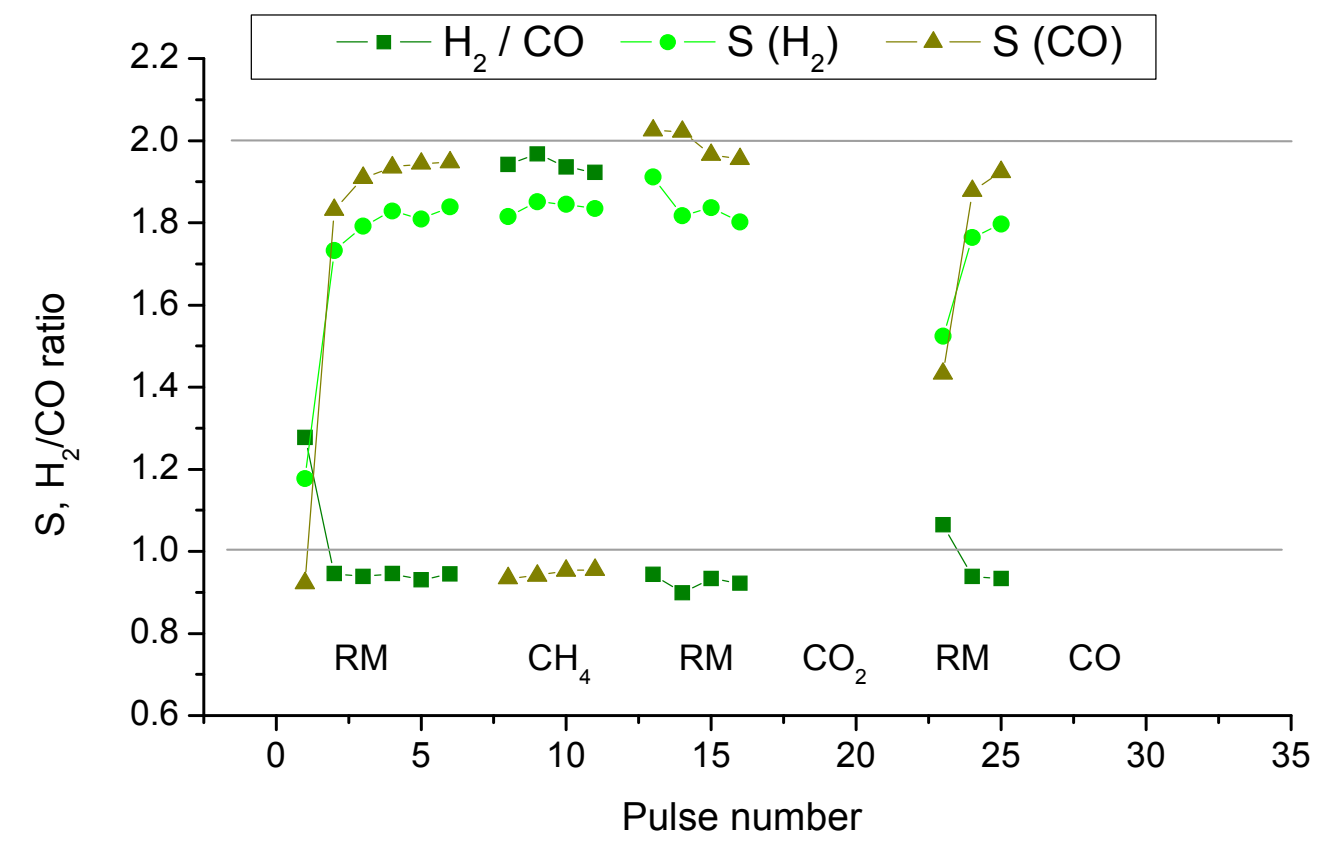

Figure 2. Product selectivities and syngas composition in series of pulses at $973 \mathrm{~K}$.

Figure 3 shows the values of the measured heat effects depending on the pulse. It is clearly seen that when the pulses of $\mathrm{RM}\left(\mathrm{CH}_{4}+\mathrm{CO}_{2}\right)$ are fed to the stationary-state catalyst, the observed heat effects are identical to the standard value of the enthalpy of methane dry reforming reaction $\sim 260 \mathrm{~kJ} / \mathrm{mol}$. In a 
series of methane pulses (pulses from 8 to 11), the heat effect shifts even more to the endothermic region, which is explained by the involvement of mobile oxygen of the catalyst. Estimated values of the oxygen bonding strength (heat of $\mathrm{O}_{2}$ chemisorption) are $\sim 655 \mathrm{~kJ} / \mathrm{mol} \mathrm{O}_{2}$.

When $\mathrm{CO}_{2}$ pulses were supplied to the stationary-state catalyst, a pronounced exothermic effect was observed due to reoxidation of the catalyst regenerating the surface oxygen species. The bonding strength of these oxygen species estimated from these experiments is $\sim 650 \mathrm{~kJ} / \mathrm{mol} \mathrm{O}{ }_{2}$, which is consistent with the value obtained above. Moreover, when CO pulses were applied to the catalyst in a stationary state, a partial reduction of ceria-zirconia surface took place with CO conversion less than $10 \%$ in each pulse, and the bonding strength of oxygen species was found to be $630-670 \mathrm{~kJ} / \mathrm{mol} \mathrm{O}_{2}$.

Since conversions of reagents in their mixed and separate pulses are very close, this suggests that mechanism of $\mathrm{CH}_{4}$ dry reforming on the catalyst with a high oxygen mobility can be described by the redox scheme, in which activation of methane and carbon dioxide occurs independently.

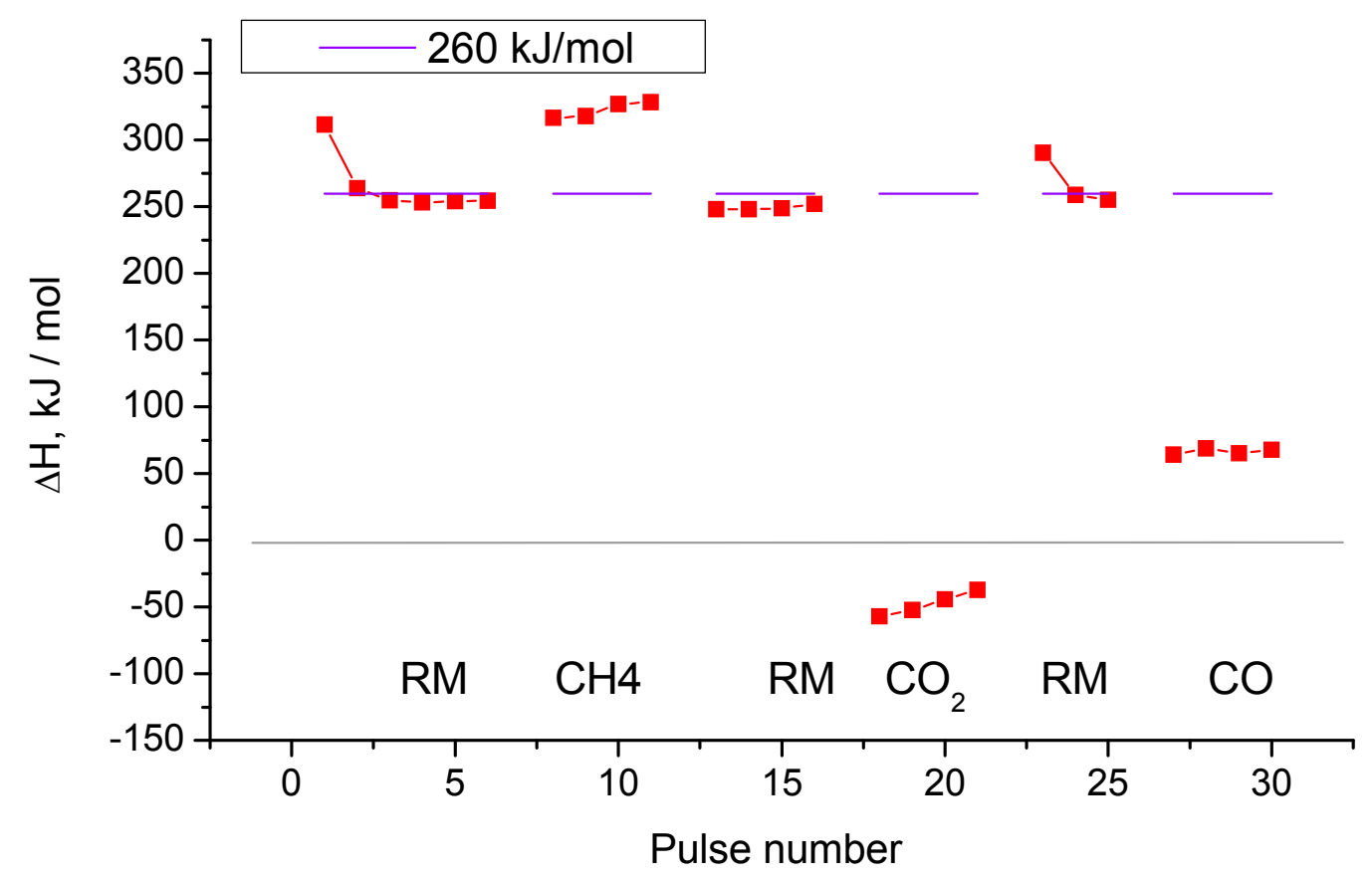

Figure 3. Measured heat effects during pulses of different compositions at $973 \mathrm{~K}$.

\subsection{Pulse Microcalorimetry at $773 \mathrm{~K}$}

The next stage of this research was the study of the reactions taking place in the system with incomplete conversion of reagents. It is well known that a decrease in temperature or contact time promotes a decrease in conversion. So, the experimental results below were obtained at $773 \mathrm{~K}$. The general procedure was as follows:

(1) Continuous flow of reaction mixture $\left(5 \% \mathrm{CH}_{4}\right.$ and $\left.\mathrm{CO}_{2}\right)$ in $\mathrm{He}$ at $773 \mathrm{~K}$ for $30 \mathrm{~min}$. After that small (pulse volume $0.85 \mathrm{~mL}$ ) probing pulses and big (pulse volume $3.6 \mathrm{~mL}$ ) pulses of reaction mixture were fed into the reactor by turns. Analysis method was the same as at $973 \mathrm{~K}$ described above.

(2) Pulses of $5 \% \mathrm{CO}_{2}$ in $\mathrm{He}$.

(3) Small (pulse volume $0.85 \mathrm{~mL}$ ) probing pulses and big (pulse volume $3.6 \mathrm{~mL}$ ) pulses of the reaction mixture.

(4) Pulses of $5 \% \mathrm{CH}_{4}$ in $\mathrm{He}$ (pulse volume $0.85 \mathrm{~mL}$ ).

(5) Pulses of $5 \% \mathrm{CO}_{2}$ in $\mathrm{He}$.

Figures 4 and 5 show conversions of methane and $\mathrm{CO}_{2}$, the mass balance as well as the ratios of hydrogen and $\mathrm{CO}$ including selectivities defined as mole of products per mole of converted methane 
calculated from the chromatographic analysis data. It should be noted that, like at a higher temperature, the mass balance in the entire experiment was close to unity, which indicates the reliability of the data obtained. During the first pulse of the RM, there was an excessive release of $\mathrm{H}_{2}$ and $\mathrm{CO}_{2}$, associated with the participation of the oxygen of the catalyst, being in a more oxidized state compared with the stationary state of the catalyst. Starting with the 2nd pulse of the RM, conversions of reagents are stabilized. Further, $\mathrm{CO}_{2}$ pulses were fed to the catalyst in a steady state, during which a gradual decrease in the degree of conversion of $\mathrm{CO}_{2}$ was observed due to the reoxidation of the steady-state catalyst.

After that, the catalyst was again brought to a steady state and methane pulses were fed in the absence of $\mathrm{CO}_{2}$, while the synthesis gas was released, but the ratio of $\mathrm{H}_{2}$ to $\mathrm{CO}$ was about 2.4 with a gradual decrease to 2 with a lowering methane conversion from $60 \%$ to $50 \%$, which is associated with the reaction of formation of the synthesis gas with the participation of the catalyst oxygen $\mathrm{O}_{\text {cat }}$ in accordance with Equation (2). The decrease in methane conversion is associated with the depletion of the catalyst mobile oxygen $\mathrm{O}_{\text {cat }}$.

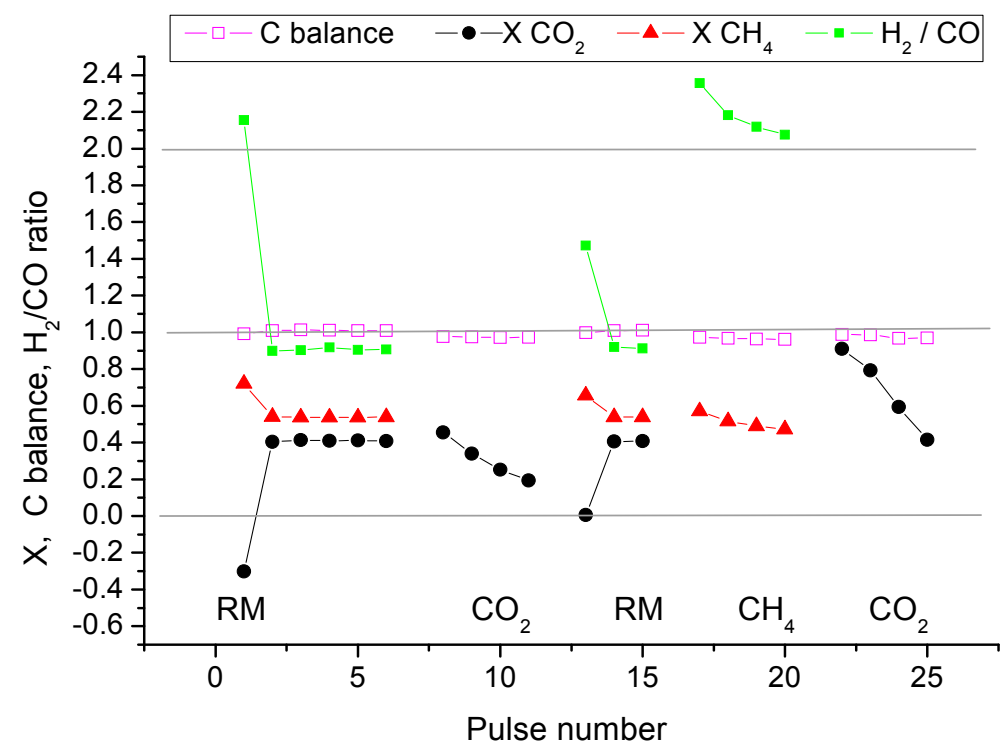

Figure 4. Conversions of reagents and products characteristics in series of pulses at $773 \mathrm{~K}$.

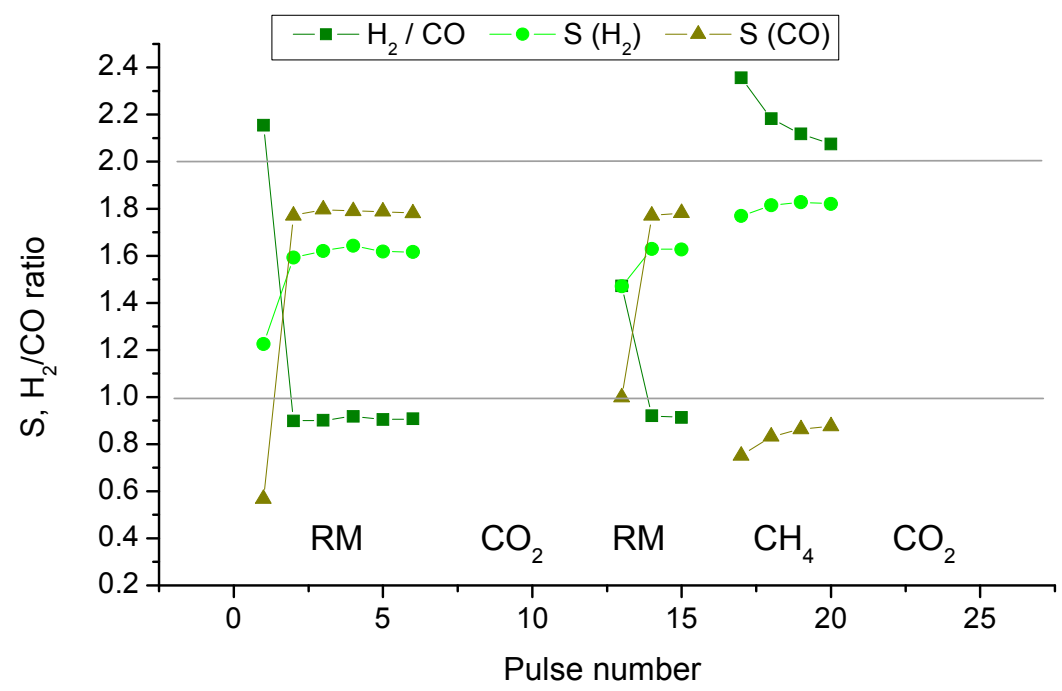

Figure 5. Product selectivities and syngas composition in series of pulses at $773 \mathrm{~K}$. 
Figure 6 shows the values of the measured heat effects depending on the pulse composition. The data obtained with incomplete conversions of reagents are consistent with those obtained previously at full conversion. Thus, when pulses of the $\mathrm{RM}\left(\mathrm{CH}_{4}+\mathrm{CO}_{2}\right)$ are fed to the catalyst in the stationary state, the observed heat effects are identical to the standard value of the enthalpy of methane dry reforming reaction (1), about $260 \mathrm{~kJ} / \mathrm{mol}$. The exothermic effect when $\mathrm{CO}_{2}$ pulses are fed to the stationary catalyst is due to its reoxidation, the surface oxygen bonding strength is $650 \mathrm{~kJ}$ per mol $\mathrm{O}_{2}$, which agrees with the values obtained above. In a series of methane pulses (pulses from 16 to 19), the thermal effect shifts to the endothermic region in comparison with the tabulated value of methane dry reforming reaction, which is explained by participation of the surface oxygen yielding synthesis gas according to reaction (2). The heat of oxygen chemisorption for the steady-state catalyst determined from the measured heat effects at $773 \mathrm{~K}$, was about $650 \mathrm{~kJ}$ per mol $\mathrm{O}_{2}$. All the observed regularities were similar for the results obtained at $823 \mathrm{~K}$, which are not shown for brevity.

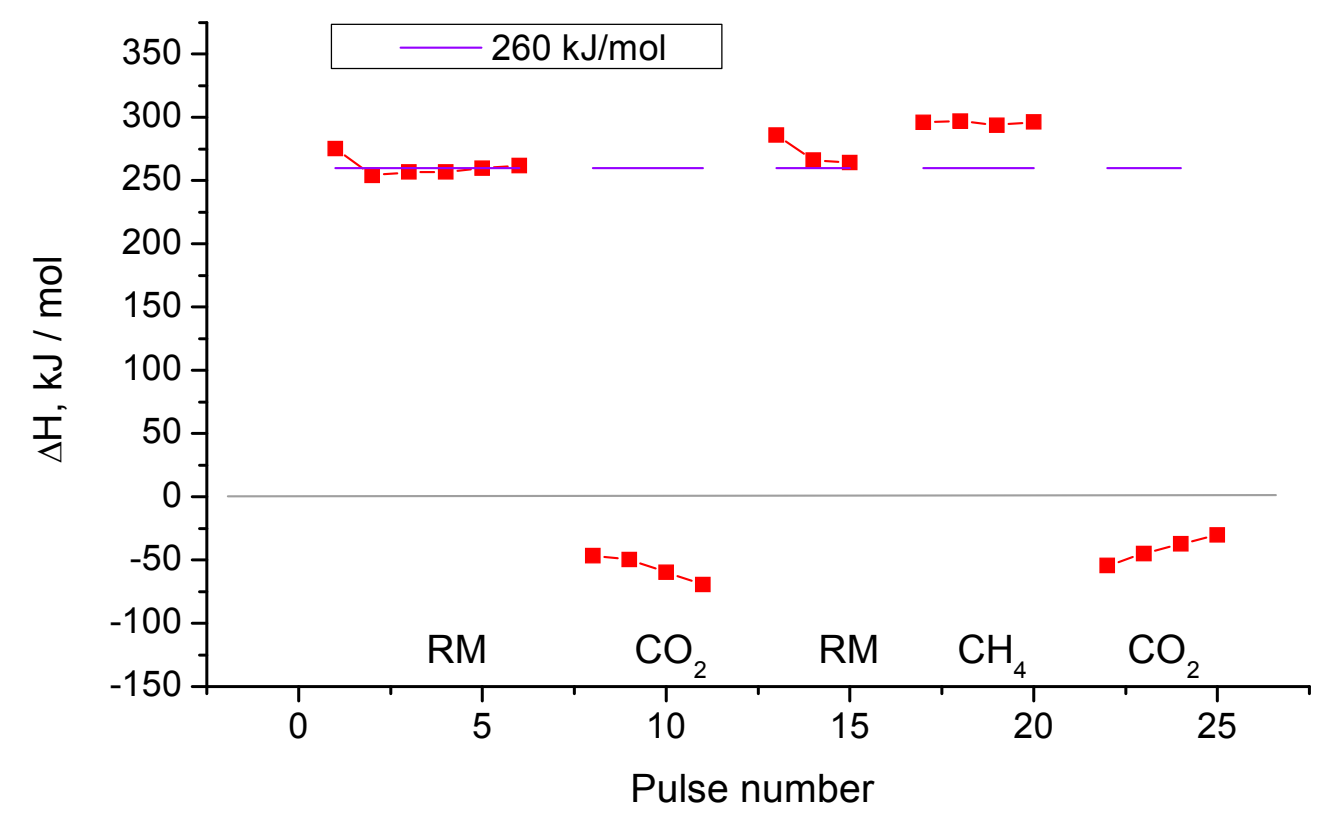

Figure 6. Measured heat effects during pulses of different compositions at $773 \mathrm{~K}$.

As to the comparison of the results obtained at 973 and $773 \mathrm{~K}$, the differences obtained in these two series of experiments consist mainly in conversions of reagents and in $\mathrm{H}_{2} / \mathrm{CO}$ ratio which is caused by the temperature dependence of WGS reaction. The measured values of the thermal effects of chemisorption of oxygen coincide with each other and with the data obtained earlier for fluorite-like and perovskite-like catalysts [13].

Additionally, experiments were performed with a modified sequence of pulses. As shown in Figure 7, after achieving the steady-state with $\mathrm{RM}$ pulses, $\mathrm{CO}_{2}$ pulses were applied followed by methane pulses. There were no pulses of the reaction mixture between pulses of $\mathrm{CO}_{2}$ and methane. The $\mathrm{CO}_{2}$ conversion in $\mathrm{CO}_{2}$ pulses is slowly decreasing, which is due to the oxidation of the stationary catalyst. When methane pulses are fed, its conversion firstly reaches 0.8 and gradually decreases below the stationary level, which is associated with the consumption of mobile oxygen of cerium-zirconium oxide. 


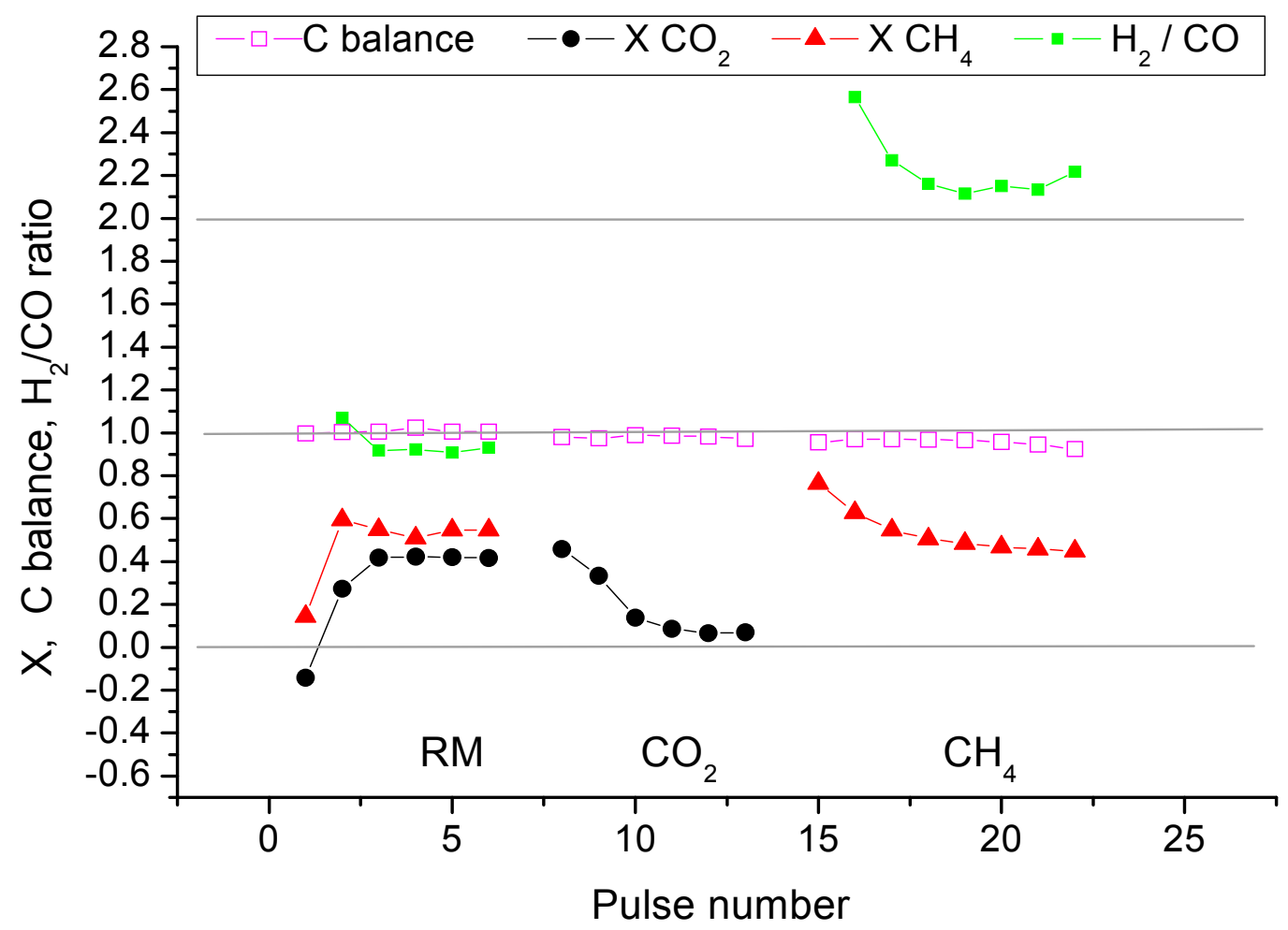

Figure 7. Conversions of reagents and products characteristics in series of pulses at $773 \mathrm{~K}$.

As can be seen from Figure 8, the amount of hydrogen is approximately equal to the amount of $\mathrm{CO}$ in terms of converted methane in the case of RM pulses, which corresponds to the stoichiometry of the methane dry reforming reaction. During $\mathrm{RM}$ pulses the amount of $\mathrm{H}_{2}$ is slightly less than $\mathrm{CO}$ because of the reverse water gas shift reaction. In methane pulses, the ratio of hydrogen to $\mathrm{CO}$ is much higher exceeding 2, which is explained by the oxidation reaction of methane with mobile oxygen of the catalyst. In this case, the quantity of products tends to decrease after the twentieth pulse, which is due to the consumption of the reactive/mobile oxygen species.

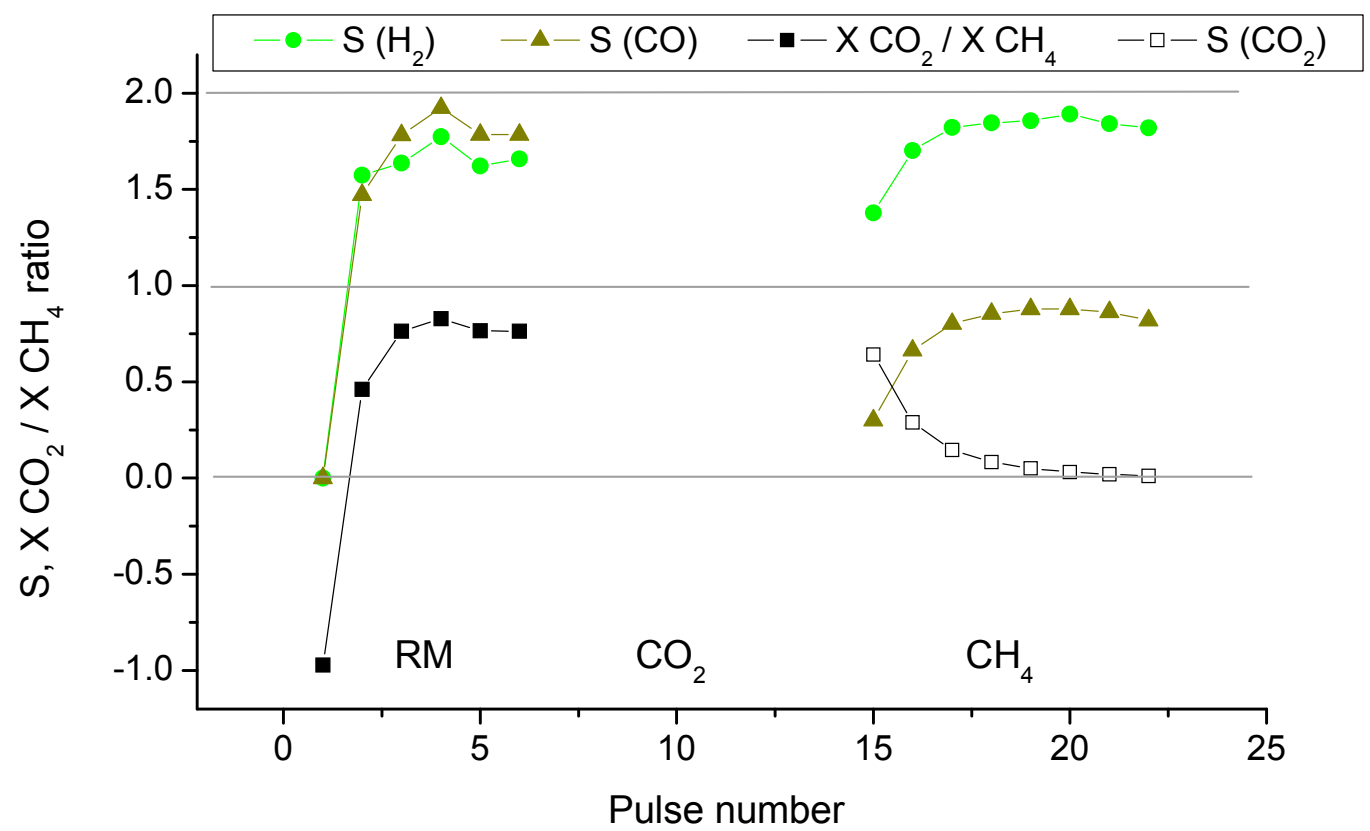

Figure 8. Product selectivities and conversions ratio in a series of pulses at $773 \mathrm{~K}$. 
Figure 9 shows the heat effects for this series of experiments. It is clear that the heat effect when the RM pulses are fed is exactly equal to the standard enthalpy of methane dry reforming reaction. However, when feeding methane pulses, the thermal effect is more endothermic not depending on the degree of methane conversion, which is due to participation of the catalyst's oxygen.

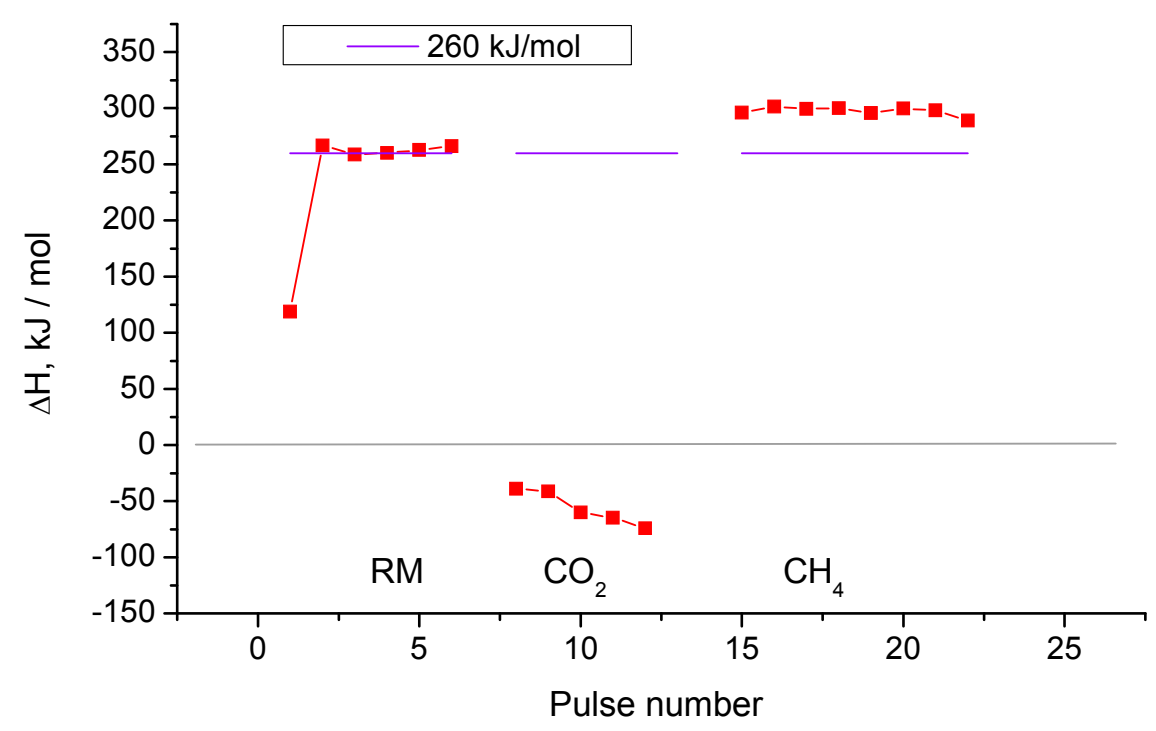

Figure 9. Measured heat effects during pulses of different compositions at $773 \mathrm{~K}$.

Experiments were also carried out to oxidize the steady-state catalyst with pulses of oxygen in He. As can be seen from Figure 10, oxygen was completely absorbed by feeding seven pulses with a volume of $0.85 \mathrm{~mL}$. To accelerate the oxidation process, two large pulses with a volume of $3.6 \mathrm{~mL}$ were then fed, the first of which was completely absorbed. In the course of subsequent pulses, the oxygen uptake gradually decreased to zero. The total amount of chemisorbed oxygen was $2.85 \times 10^{-5}$ moles $\mathrm{O}_{2}$ or 11.2 monolayers indicating participation of sub-surface oxygen. The thermal exo effect is approximately constant and does not depend on the volume of the pulse, as can be seen from Figure 11.

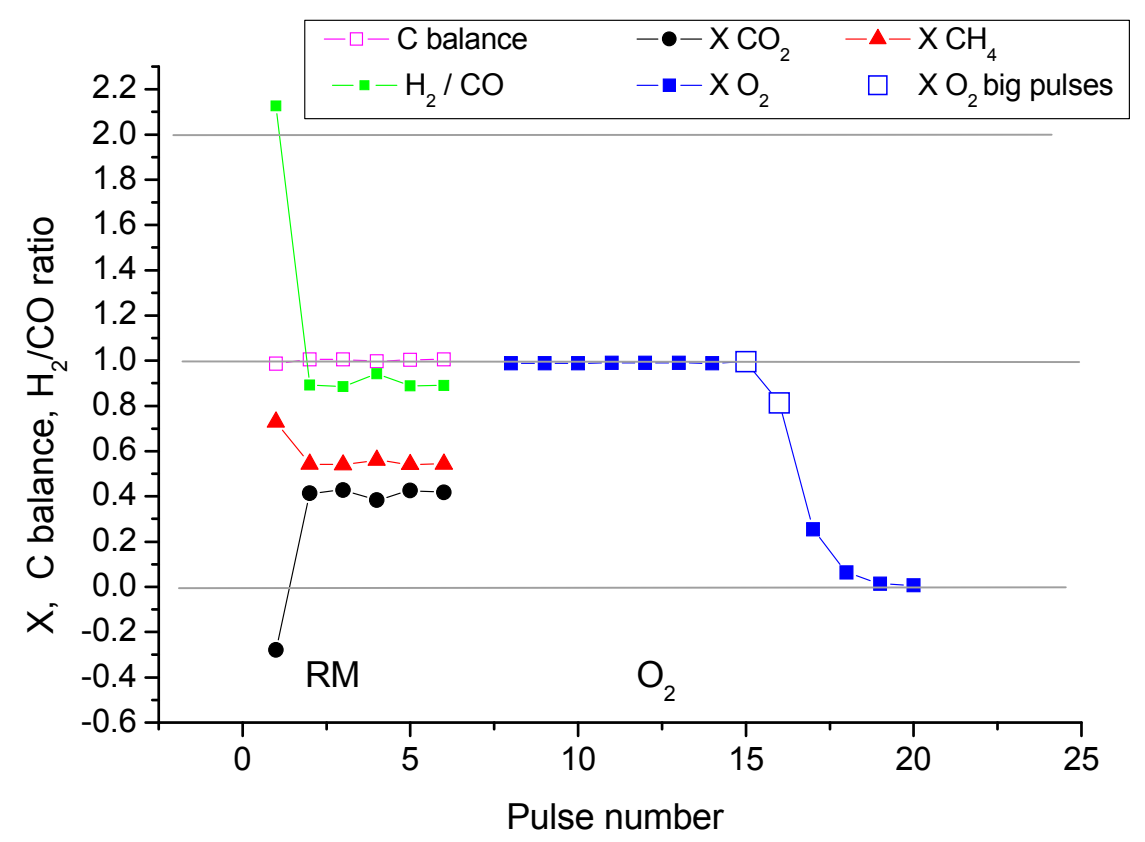

Figure 10. Conversions of reagents and product characteristics in series of the reaction mixture pulses followed by $\mathrm{O}_{2}$ pulses at $773 \mathrm{~K}$. 


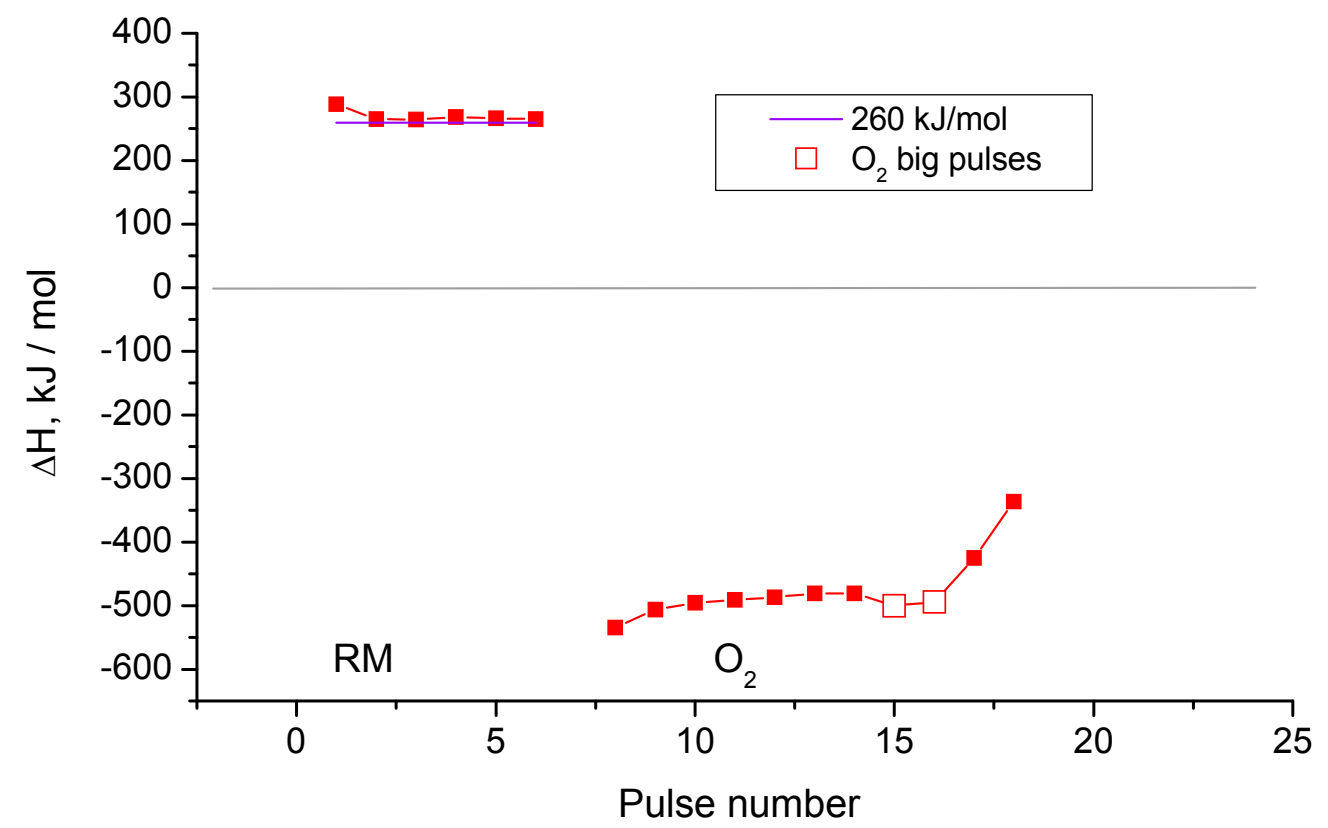

Figure 11. Measured heat effects for the series of the reaction mixture pulses followed by $\mathrm{O}_{2}$ pulses at $773 \mathrm{~K}$.

\section{Materials and Methods}

A continuous preparation technique in supercritical alcohol medium was employed for the preparation of $\mathrm{Ni}$ /ceria-zirconia catalysts with controllable particle size of the mixed oxide support and nickel dispersion [14]. The average particle sizes of ceria-zirconia and nickel oxide were estimated by the Selyakov-Scherrer equation from the integral broadening of the most intensive (002) and (200) XRD (X-ray diffraction) peaks, respectively. Transmission electron microscopy (TEM) micrographs were obtained with a JEM-2010 instrument (JEOL Ltd., Tokyo, Japan). The nickel $(\mathrm{NiO})$ particle size was estimated by measuring the size of at least 150 particles. Rates and heats of the catalysts reduction/reoxidation by pulses of $\mathrm{CH}_{4} / \mathrm{CO}_{2} / \mathrm{O}_{2}$ in He for catalysts after different pretreatment were estimated using a Setaram Sensys DSC TG calorimeter (Setaram Instrumentation, Caluire-et-Cuire, France) and a pulse kinetic installation equipped with gas chromatograph Chromos GH-1000 (Chromosib, Moscow, Russia), mass-spectrometer UGA-200 (Stanford Research Systems Inc., Sunnyvale, CA, USA), and gas sensors. The catalyst sample $(0.033 \mathrm{~g})$ was loaded in a quartz tube which was located in a measuring channel of the calorimetric unit. In the comparison channel an empty reactor was placed. Inlet gas feed was produced by the gas-mixing installation and pulses were introduced into the reactor by a six-port valve. The catalyst steady state was achieved using a continuous flow of the reaction feed followed by purging reactor with the He stream for $30 \mathrm{~min}$ and supplying several consecutive pulses of the reaction mixture at certain temperature, namely 773 and $973 \mathrm{~K}$. The volume of each pulse was equal to $0.85 \mathrm{~mL}$ or $3.6 \mathrm{~mL}$, the time interval between pulses $20 \mathrm{~min}$. Further details are described elsewhere [13].

Conversion of reagents $X$ was estimated as follows:

$$
\begin{aligned}
& X_{\mathrm{CH} 4}=\frac{C_{\mathrm{CH} 4}}{C_{\mathrm{CH} 4}^{0}-C_{\mathrm{CH} 4}} \\
& X_{\mathrm{CO} 2}=\frac{C_{\mathrm{CO} 2}}{C_{\mathrm{CO} 2}^{0}-C_{\mathrm{CO} 2}}
\end{aligned}
$$


and selectivity $S$ in the current work is defined as

$$
\begin{aligned}
S_{\mathrm{H} 2} & =\frac{C_{\mathrm{H} 2}}{C_{\mathrm{CH} 4}^{0}-C_{\mathrm{CH} 4}} \\
S_{\mathrm{CO}} & =\frac{C_{\mathrm{CO}}}{C_{\mathrm{CH} 4}^{0}-C_{\mathrm{CH} 4}} \\
S_{\mathrm{CO} 2} & =\frac{C_{\mathrm{CO} 2}}{C_{\mathrm{CH} 4}^{0}-C_{\mathrm{CH} 4}}
\end{aligned}
$$

where $C_{i}$ is concentration of certain component in products mixture, $C_{i}{ }^{0}$ initial concentration, $S_{\mathrm{CO} 2}$ was calculated in the case of $\mathrm{CH}_{4}$ pulses only.

The amount of oxygen removed from the catalyst by $\mathrm{CH}_{4}$ pulse or inserted by $\mathrm{CO}_{2} / \mathrm{O}_{2}$ pulse was expressed either as a fraction of the total amount of oxygen in oxidized sample or a number of the oxygen monolayers (one monolayer is equal to $10^{19}$ atoms $\mathrm{m}^{-2}$ ).

\section{Conclusions}

The studied process of methane dry reforming can be described by a sequential redox scheme and the activation of methane and carbon dioxide occurs independently. Oxidation of methane with release of the synthesis gas components is accompanied by a partial reduction of the catalyst, while reoxidation of the sample with carbon dioxide is a necessary stage for the replenishment of mobile oxygen. Calculated values of the heat of oxygen chemisorption during feeding pulses of $\mathrm{CH}_{4}, \mathrm{CO}_{2}$, or $\mathrm{CO}$ reliably agree with each other and amount to about $650 \mathrm{~kJ}$ per $\mathrm{mol} \mathrm{O}_{2}$, which corresponds to bridging oxygen forms.

Acknowledgments: The work was carried out with the financial support of the Ministry of Education and Science of the Russian Federation under the Agreement 14.616.21.0036, unique agreement ID RFMEFI61615X0036.

Author Contributions: M.Y.S. prepared and characterized sample for studies; V.A.S. and V.A.R planned and designed microcalorimetric experiments; V.A.R and M.N.S. performed experiments and analyzed the data; M.N.S. and V.A.S wrote the paper.

Conflicts of Interest: The authors declare no conflict of interest. The founding sponsors had no role in the design of the study; in the collection, analyses, or interpretation of data; in the writing of the manuscript, and in the decision to publish the results.

\section{References}

1. Koubaissy, B.; Pietraszek, A.; Roger, A.C.; Kiennemann, A. $\mathrm{CO}_{2}$ reforming of methane over Ce-Zr-Ni-Me mixed catalysts. Catal. Today 2010, 157, 436-439. [CrossRef]

2. Kambolis, A.; Matralis, H.; Trovarelli, A.; Papadopoulou, Ch. Ni/CeO $-\mathrm{ZrO}_{2}$ catalysts for the dry reforming of methane. Appl. Catal. A 2010, 377, 16-26. [CrossRef]

3. Damyanova, S.; Pawelec, B.; Arishtirova, K.; Huerta, M.V.M.; Fierro, J.L.G. The effect of $\mathrm{CeO}_{2}$ on the surface and catalytic properties of $\mathrm{Pt} / \mathrm{CeO}_{2}-\mathrm{ZrO}_{2}$ catalysts for methane dry reforming. Appl. Catal. B 2009, 89, 149-159. [CrossRef]

4. Sadykov, V.A.; Gubanova, E.L.; Sazonova, N.N.; Pokrovskaya, S.A.; Chumakova, N.A.; Mezentseva, N.V.; Bobin, A.S.; Gulyaev, R.V.; Ishchenko, A.V.; Krieger, T.A.; et al. Dry reforming of methane over Pt/PrCeZrO catalyst: Kinetic and mechanistic features by transient studies and their modeling. Catal. Today 2011, 171, 140-149. [CrossRef]

5. Pavlova, S.N.; Sazonova, N.N.; Sadykov, V.A.; Alikina, G.M.; Lukashevich, A.I.; Gubanova, E.L.; Bunina, R.V. Study of synthesis gas production over structured catalysts based on $\mathrm{LaNi}(\mathrm{Pt}) \mathrm{O}_{\mathrm{x}}-$ and $\mathrm{Pt}(\mathrm{LaPt})-\mathrm{CeO}_{2}-\mathrm{ZrO}_{2}$ supported on corundum. Stud. Surf. Sci. Catal. 2007, 167, 343-348.

6. Bitter, J.H.; Seshan, K.; Lercher, J.A. Mono and bifunctional pathways of $\mathrm{CO}_{2} / \mathrm{CH}_{4}$ reforming over Pt and $\mathrm{Rh}$ based catalysts. J. Catal. 1998, 176, 93-101. [CrossRef] 
7. Bychkov, V.; Tyulenin, Yu.; Krylov, O.; Korchak, V. Methane reforming with carbon dioxide on the $\mathrm{Co} /-\mathrm{Al}_{2} \mathrm{O}_{3}$ catalyst: The formation, state, and transformations of surface carbon. Kinet. Catal. 2002, 43, 724-730. [CrossRef]

8. Bychkov, V.; Tyulenin, Yu.; Krylov, O.; Korchak, V. The mechanism of methane reforming with carbon dioxide: Comparison of supported Pt and $\mathrm{Ni}$ (Co) catalysts. Kinet. Catal. 2003, 44, 353-359. [CrossRef]

9. Schuurman, Y.; Mirodatos, C. Uses of transient kinetics for methane activation studies. Appl. Catal. A 1997, 151, 305-331. [CrossRef]

10. Slagtern, Y.; Schuurman, C.; Leclercq, X.; Verykios, C. Mirodatos, Specific features concerning the mechanism of methane reforming by carbon dioxide over $\mathrm{Ni} / \mathrm{La}_{2} \mathrm{O}_{3}$ catalyst. J. Catal. 1997, 172, 118-126. [CrossRef]

11. Pavlova, S.N.; Sadykov, V.A.; Bulgakov, N.N.; Bredikhin, M.N. The influence of support on the low-temperature activity of Pd in the reaction of CO oxidation: 3. Kinetics and mechanism of the reaction. J. Catal. 1996, 161, 517-523. [CrossRef]

12. Sadykov, V.A.; Tikhov, S.F.; Bulgakov, N.N.; Gerasev, A.P. Catalytic oxidation of $\mathrm{CO}$ on $\mathrm{CuO}_{\mathrm{x}}$ revisited: Impact of the surface state on the apparent kinetic parameters. Catal. Today 2009, 144, 324-333. [CrossRef]

13. Sadykov, V.; Rogov, V.; Ermakova, E.; Arendarsky, D.; Mezentseva, N.; Alikina, G.; Sazonova, N.; Bobin, A.; Pavlova, S.; Schuurman, Y.; et al. Mechanism of $\mathrm{CH}_{4}$ dry reforming by pulse microcalorimetry: Metal nanoparticles on perovskite/fluorite supports with high oxygen mobility. Thermochim. Acta 2013, 567, 27-34. [CrossRef]

14. Smirnova, M.Y.; Pavlova, S.N.; Krieger, T.A.; Bespalko, Y.N.; Anikeev, V.I.; Chesalov, Y.A.; Kaichev, V.V.; Mezentseva, N.V.; Sadykov, V.A. Synthesis of $\mathrm{Ce}_{1-\mathrm{x}} \mathrm{Zr}_{\mathrm{x}} \mathrm{O}_{2}$ oxides in supercritical alcohols and catalysts of methane dry reforming on their bases. Supercritical Fluids Theory Pract. 2017, in press.

(C) 2017 by the authors. Licensee MDPI, Basel, Switzerland. This article is an open access article distributed under the terms and conditions of the Creative Commons Attribution (CC BY) license (http:/ / creativecommons.org/licenses/by/4.0/). 